\title{
APPLICATION DU NITRATE DE CELLULOSE A LA DOSIMETRIE DES NEUTRONS RAPIDES
}

\author{
A. DRAGU*, M. NICOLAE* \\ (Manuscrit rę̧u le 2 février 1972)
}

\begin{abstract}
RÉSUMÉ
Les détecteurs solides de traces présentent des caractéristiques qui permettent de les utiliser pour la dosimétrie des neutrons rapides.

Les deux principaux types d'interaction utilisés pour la détection des neutrons sont examinés. La diffusion élastique des neutrons par le carbone, l'azote et l'oxygène permet la détection des noyaux de recul. Le rendement théorique, pour une source de $\mathrm{Pu}-\mathrm{Be}$ est de $10^{-5}$ trace par neutron. Le second type d'interaction comprend les réactions $(n, \alpha)$ sur les éléments précédents. Pour le même spectre, le rendement théorique est de $8 \cdot 10^{-6}$ trace par neutron.

Les résultats expérimentaux sont en assez bonne concordance avec les calculs théoriques pour qu'on puisse envisager l'utilisation du nitrate de cellulose comme détecteur de neutrons rapides d'énergie inférieure à $10 \mathrm{MeV}$, auprès des sources usuelles dont le spectre est connu ou peut être supposé.
\end{abstract}

\begin{abstract}
Due to the fact that solid state track detectors have some advantages compared with nuclear emulsions, they seem to be useful for fast neutron dosimetry.

The two main processes useful in neutron detection are examined. The elastic diffusion of neutrons by the carbon, nitrogen and oxygen nuclei allows the recording of the recoil nuclei. The efficiency for a Pu-Be source is $10^{-5}$ track per neutron. The second important process which is taken into account is the $(n, \alpha)$ reaction with the $\mathrm{C}, \mathrm{N}, \mathrm{O}$ nuclei. For the same spectrum the computed efficiency is $8.10^{-6}$ track per neutron.

The experimental results are in sufficiently good agreement with the theoritical computations to consider the cellulose nitrate detectors as a good fast neutron integrating device, for energies below ro $\mathrm{MeV}$ and for usual neutron sources of known spectrum.
\end{abstract}

\section{I - INTRODUCTION}

L'utilisation des détecteurs solides de traces pour la dosimétrie des neutrons rapides est mentionnée très souvent parmi les applications possibles de cette classe de détecteurs. Une série de résultats a déjà été présentée concernant l'utilisation du makrofol et des polycarbonates pour la mesure de la fluence des neutrons rapides $[1,2,3]$.

* Institut de Physique Atomique - Bucarest (Roumanie).

RADIOPROTECTION, VOL. 7 - $\mathrm{N}^{\circ} 2$ 
Il semble qu'un dosimètre personnel pour neutrons rapides de ce type pourrait présenter certains avantages par rapport aux dosimètres à émulsions nucléaires. La sensibilité et la stabilité du détecteur solide sont moins affectées par les facteurs environnants que celles du film photographique. Les détecteurs solides de traces sont également plus commodes et plus simples à traiter et à interpréter.

En raison des considérations ci-dessus, nous nous sommes proposé d'effectuer une étude systématique concernant l'emploi du nitrate de cellulose pour la dosimétrie des neutrons rapides. A cet effet, nous avons étudié :

I) le principe de la méthode la plus convenable,

2) la limite théorique de la sensibilité de la méthode,

3) les caractéristiques d'enregistrement du détecteur,

4) l'application du détecteur de nitrate de cellulose à la dosimétrie des neutrons rapides,

5) les conclusions concernant l'application du nitrate de cellulose à la dosimétrie des neutrons rapides et sa comparaison avec les émulsions nucléaires.

\section{2 - PRINCIPE DE LA MÉTHODE}

L'enregistrement des particules ionisantes dans les détecteurs solides de traces devient possible si la perte d'énergie de la particule, par unité de parcours, est suffisamment élevée pour produire des modifications de structure dans la molécule du polymère, en particulier la dégradation de celle-ci de façon que la révélation chimique du défaut créé soit possible. Les différences de sensibilité des polymères synthétiques, en ce qui concerne l'enregistrement des particules ionisantes, sont dues à leurs particularités de structure; parmi celles-ci un rôle très important est joué par le nombre des liaisons chimiques susceptibles d'être détruites par unité de parcours de la particule.

De ce point de vue, le nitrate de cellulose possède, jusqu'à présent, la sensibilité la plus élevée parmi les détecteurs plastiques.

Le nitrate de cellulose est couramment utilisé comme détecteur de neutrons rapides car il est constitué d'ions légers (carbone, oxygène, azote) qui peuvent interagir avec les neutrons rapides en donnant des particules chargées enregistrables par le détecteur.

Lors de l'interaction des neutrons rapides avec les atomes mentionnés ci-dessus, deux types de phénomènes sont intéressants : la diffusion des neutrons sur les ions légers (carbone, oxygène, azote) et les réactions du type $(n, \alpha)$ sur les mêmes noyaux. Les ions de recul résultant de la diffusion élastique (sauf pour l'hydrogène) ainsi que les particules $\alpha$ sont susceptibles d'être enregistrés par le nitrate de cellulose. L'analyse de la variation des paramètres de ces processus nucléaires en fonction de l'énergie des neutrons rapides peut nous fournir une série de renseignements sur le seuil théorique de la sensibilité de la méthode et sur les limites de son utilisation. Dans ce but, nous avons analysé séparément les deux types d'interactions qui peuvent donner, après une attaque convenable, des traces visibles. En mesurant la densité de traces dans l'unité de surface du détecteur, la fluence de neutrons et la dose absorbée peuvent être déterminées. 


\section{3 - SENSIBILITÉ DE LA MÉTHODE}

\section{I. Diffusion Élastique Des neutrons RAPIDES PAR LES ATOMES DE CARBone, OXYGÈNE, AZOTE}

Dans ce travail, on n'a considéré que la diffusion élastique. En effet, la diffusion inélastique ne devient importante que pour des énergies de neutrons beaucoup plus élevées que celles des sources usuelles.

Pour l'utilisation de cette méthode, les caractéristiques suivantes nous intéressent :

I) l'énergie des ions de recul, $\mathrm{C}, \mathrm{N}, \mathrm{O}$, obtenus par diffusion élastique des neutrons rapides d'énergie variable et la perte linéique d'énergie de ces ions au début de leur parcours,

2) les parcours des ions de recul dans le détecteur en fonction de l'énergie,

3) le nombre total d'ions de recul obtenus par unité de surface du détecteur.

Pour trouver les énergies des ions de recul, on a utilisé la loi de conservation de l'énergie cinétique et de l'impulsion, dans le système du laboratoire (énergies non relativistes) $[4,5]$.

$$
\begin{aligned}
E_{n} & =E_{n}^{\prime}+E \\
\sqrt{2 M_{n} E_{n}} & =\sqrt{2 M_{n} E_{n}^{\prime}} \cos \gamma+\sqrt{2 M_{i} E_{i}} \cos \varphi \\
\mathrm{O} & =\sqrt{2 M_{n} E_{n}^{\prime}} \sin \gamma-2 M_{i} E_{i} \sin \varphi
\end{aligned}
$$

où

$E_{n}=$ énergie initiale du neutron

$E^{\prime}{ }_{n}=$ énergie du neutron après impact

$M_{n}=$ masse du neutron

$E_{i}=$ énergie cinétique de l'ion de recul

$M_{i}=$ masse de l'ion de recul

$\gamma=$ angle de diffusion du neutron avec sa direction initiale

$\varphi=$ angle de la direction de l'ion de recul avec la direction initiale du neutron.

A partir des relations ci-dessus, on obtient l'énergie moyenne de l'ion de recul :

$$
\bar{E}_{i}=\frac{2 M_{n} M_{i}}{\left(M_{n}+M_{i}\right)^{2}} \cdot E_{n}
$$

Dans le tableau I sont données les valeurs calculées à l'aide de cette relation, pour des énergies de neutrons comprises entre 4 et $40 \mathrm{MeV}$. Pour une énergie de neutrons rapides de $4 \circ \mathrm{MeV}$, l'énergie moyenne des ions de recul est de 5,68 MeV pour le carbone, $4,98 \mathrm{MeV}$ pour l'azote et $4,43 \mathrm{MeV}$ pour l'oxygène, donc s $\mathrm{MeV}$ environ pour tous ces ions.

vol. $7-\mathrm{N}^{\mathrm{O}} 2$ 
TABLEAU I

PARAMÈTRES CALCULÉS DE LA DIFFUSION ÉLASTIQUE DES NEUTRONS RAPIDES SUR LES NOYAUX DE $\mathrm{C}, \mathrm{N}, \mathrm{O}$

\begin{tabular}{|c|c|c|c|c|c|c|c|c|c|c|c|c|}
\hline \multicolumn{5}{|c|}{ Carbone } & \multicolumn{4}{|c|}{ Azote } & \multicolumn{4}{|c|}{ Oxygène } \\
\hline $\begin{array}{c}E_{n} \\
(\mathrm{MeV})\end{array}$ & $\begin{array}{c}\bar{E}_{C} \\
(\mathrm{MeV})\end{array}$ & $\begin{array}{c}\frac{\mathrm{d} E_{C}}{\mathrm{~d} x} \\
\left(\frac{\mathrm{MeV}}{\mathrm{mg} / \mathrm{cm}^{2}}\right)\end{array}$ & $\begin{array}{c}R_{C} \\
(\mu \mathrm{m})\end{array}$ & $\begin{array}{c}\sigma_{e l}{ }_{C} \\
(b)\end{array}$ & $\begin{array}{c}\bar{E}_{N} \\
(\mathrm{MeV})\end{array}$ & $\begin{array}{c}\frac{\mathrm{d} E_{N}}{\mathrm{~d} x} \\
\left(\frac{\mathrm{MeV}}{\mathrm{mg} / \mathrm{cm}^{2}}\right)\end{array}$ & $\begin{array}{c}\mathrm{R}_{N} \\
(\mu \mathrm{m})\end{array}$ & $\begin{array}{c}\sigma_{e l}{ }_{N} \\
\text { (barn) }\end{array}$ & $\begin{array}{c}\overline{E_{O}} \\
(\mathrm{MeV})\end{array}$ & $\begin{array}{c}\frac{\mathrm{d} E_{O}}{\mathrm{~d} x} \\
\left(\frac{\mathrm{MeV}}{\mathrm{mg} / \mathrm{cm}^{2}}\right)\end{array}$ & $\begin{array}{c}\mathrm{R}_{o} \\
(\mu \mathrm{m})\end{array}$ & $\begin{array}{l}\sigma_{e l_{o}} \\
\text { (barn) }\end{array}$ \\
\hline 4,0 & 0,57 & 0,72 & & & & & & & & & & \\
\hline 4,5 & 0,64 & 1,33 & & & & & & & & & & \\
\hline 5,0 & 0,71 & 1,82 & $\mathrm{I}, 2 \mathrm{I}$ & 1,15 & 0,62 & 0,43 & & & & & & \\
\hline 5,5 & 0,78 & 2,28 & 1,29 & 1,03 & 0,68 & 1,03 & & & & & & \\
\hline 6,0 & 0,85 & 2,65 & 1,37 & 0,87 & 0,75 & 1,52 & 1,22 & 1,00 & & & & \\
\hline 7,0 & 0,99 & 3,35 & 1,45 & 0,56 & 0,87 & 2,40 & 1,35 & 0,80 & 0,78 & $\mathrm{I}, \mathrm{II}$ & & \\
\hline 8,0 & 1,14 & 3,89 & 1,60 & 1,29 & 0,99 & 3,17 & 1,48 & 0,80 & 0,89 & 2,03 & 1,17 & $0,5 \mathrm{I}$ \\
\hline 9,0 & 1,28 & 4,36 & 1,76 & 0,74 & 1,12 & 3,87 & 1,61 & 0,87 & 1,00 & 2,82 & 1,29 & 0,78 \\
\hline 10,0 & 1,42 & 4,71 & 1,90 & 0,79 & 1,24 & 4,27 & 1,75 & 0,90 & $\mathrm{I}, \mathrm{II}$ & 3,32 & $\mathrm{I}, 4 \mathrm{I}$ & 0,77 \\
\hline 12,0 & 1,70 & 5,32 & 2,03 & * & $\mathrm{I}, 49$ & 5,10 & 1,99 & $\approx 0,96$ & 1,33 & $4,4 \mathrm{I}$ & 1,63 & * \\
\hline 15,0 & 2,13 & 5,76 & 2,29 & $\approx 0,77$ & 1,87 & 5,93 & 2,30 & $\approx 1,28$ & 1,66 & 5,58 & 1,96 & \\
\hline 20,0 & 2,84 & 6,24 & 2,66 & $*$ & 2,49 & 6,85 & 2,78 & ${ }^{*}$ & 2,21 & 6,82 & 2,42 & \\
\hline 25,0 & 3,55 & 6,44 & 3,23 & & $3, \mathrm{II}$ & 7,39 & 3,21 & & 2,77 & 7,85 & $2,8 \mathrm{I}$ & \\
\hline 30,0 & 4,26 & 6,59 & $3,7^{8}$ & & 3,73 & 7,70 & 3,62 & & 3,32 & 8,62 & 3,15 & \\
\hline 35,0 & 4,97 & 6,59 & 4,34 & & 4,35 & 7,81 & 4,04 & & 3,88 & 8,78 & 3,48 & \\
\hline 40,0 & 5,68 & 6,52 & 5,50 & & $4,9^{8}$ & 8,06 & 4,46 & & 4,43 & 9,09 & 3,82 & \\
\hline
\end{tabular}

* Pour les énergies de neutrons supérieures à ro $\mathrm{MeV}$, on n'a pas trouvé les sections efficaces de diffusion élastique, mais elles peuvent être déterminées par la relation $\sigma_{e l} \approx \frac{\sigma_{T}}{2}$ ou calculées en fonction de l'énergie des neutrons.

La perte linéique d'énergie des ions de recul en fonction de l'énergie des neutrons incidents a été calculée à l'aide de la relation donnée par FLEISCHER [7].

$$
\left(\frac{\mathrm{d} E_{i}}{\mathrm{~d} x}\right)_{\beta}=\left[Z^{*}(\beta)\right]^{2}\left(\frac{\mathrm{d} E_{p}}{\mathrm{~d} x}\right)_{\beta}
$$

où $Z^{*}$ est la charge effective de l'ion à la vitesse $v$, et $\left(\mathrm{d} E_{p} / \mathrm{d} x\right)$ la perte linéique d'énergie du proton à la même vitesse. Heckmann et collab. [6] donnent pour $Z^{*}$ une relation en accord avec une courbe expérimentale et qui peut être utilisée jusqu'à une vitesse $\beta=0,25 \cdot 10^{-2} Z^{2 / 3}$ pour calculer la charge effective des ions ayant un $Z$ compris entre 2 et 20 :

$$
Z^{*}(\beta)=Z\left[\mathrm{r}-\exp \left(-\mathrm{r} 25 \beta / Z^{2 / 3}\right)\right]
$$

$Z$ est la charge de l'ion considéré et $Z^{*}$ sa charge effective. Pour calculer le $(\mathrm{d} E / \mathrm{d} x)_{\beta}$ des ions dans le nitrate de cellulose, nous avons utilisé la relation de FLeISCHER [7] : 


$$
\left(\frac{\mathrm{d} E_{p}}{\mathrm{~d} x}\right)_{\text {cellulose }}^{\text {nitrate }}=\left[0,073 \frac{\text { In } E_{p}}{E_{p}}+\frac{0,232}{E_{p}}\right] \mathrm{MeV} \cdot \mathrm{cm}^{+2} \cdot \mathrm{mg}^{-1}
$$

où $E_{p}$ est donné en $\mathrm{MeV}$. Le $\frac{\mathrm{d} E_{i}}{\mathrm{~d} x}$ calculé pour différentes énergies de neutrons est donné dans le tableau I.

Le parcours des ions de recul dans le nitrate de cellulose a été calculé en fonction du parcours déterminé dans le makrofol par Ralarosy et coll. [8] en utilisant la relation de BragG-KLEEMANN donnant une erreur de \pm is $\%$.

$$
\frac{\mathrm{R}_{1}}{\mathrm{R}_{0}}=\frac{\varrho_{0}}{\varrho_{1}} \sqrt{\frac{A_{1}}{A_{0}}}
$$

$R_{0}$ et $R_{1}$ sont les parcours, $\varrho_{0}$ et $\varrho_{1}$ les masses volumiques, $A_{0}$ et $A_{1}$ les poids atomiques moyens du makrofol et du nitrate, respectivement.

La masse atomique moyenne du détecteur a été calculée par la relation :

$$
\sqrt{A}=\sum_{i=1}^{p} t_{i} \sqrt{A_{i}}
$$

où $f_{i}$ est la fraction atomique de l'élément ayant le poids atomique $A_{i}$. La composition élémentaire du nitrate de cellulose utilisé dans ce travail a été donnée dans [9]. En utilisant une masse volumique du nitrate de cellulose de $1,517 \mathrm{~g} / \mathrm{cm}^{3}$, on déduit que :

$$
R_{\text {nitr. }}=0,78 R_{\text {makr }}
$$

TABLEAU II

Compostrion ÉlÉmentaire dU NITrATE DE CELlULOSE

\begin{tabular}{c|c|c}
\hline & \multicolumn{2}{|c}{ Concentration } \\
\cline { 2 - 3 } & $\%$ & $\mathrm{~g} / \mathrm{cm}^{3}$ \\
\hline $\mathrm{C}$ & 25,11 & $0,38 \mathrm{1}$ \\
$\mathrm{O}$ & 60,25 & 0,915 \\
$\mathrm{~N}$ & $1 \mathrm{1}, 72$ & 0,178 \\
$\mathrm{H}$ & 2,92 & 0,043 \\
\hline & & $\varrho=1,517$ \\
\hline
\end{tabular}

Les parcours des ions de recul calculés comme ci-dessus, en fonction de l'énergie des neutrons, sont donnés dans le tableau I. Dans le même tableau ont été incluses les sections efficaces de diffusion des neutrons rapides sur les atomes de carbone, azote, oxygène d'après [10], [ 11 ] , [1 2].

On estime [7], [8], que les longueurs minimales des traces développées dans le détecteur solide doivent être de $\mathrm{I}, 5$ à $2 \mu \mathrm{m}$ pour être visibles au microscope optique avec les méthodes usuelles d'observation. En raison de cette considération,

voL. $7-\mathrm{N}^{\circ} 2$ 
et en fonction des données du tableau I, il résulte qu'il existe un seuil d'énergie minimale des neutrons au-delà duquel les trajectoires des ions deviennent observables dans le détecteur, en supposant que les conditions d'attaque ont été choisies d'une manière convenable. Comme la perte linéique d'énergie des ions, dans le cas considéré, est suffisamment élevée par rapport au seuil énergétique du détecteur, la visualisation des traces des ions dans le détecteur est pratiquement limitée par la longueur minimale des trajectoires observables. En considérant que ce seuil d'observation se situe à $\mathrm{I}, 5-2 \mu \mathrm{m}$ pour les ions de recul, seuls les neutrons ayant des énergies supérieures à $6 \mathrm{MeV}$ donnent des ions de recul de carbone observables, et ce n'est qu'aux énergies supérieures à ro $\mathrm{MeV}$ que les traces des ions de recul du carbone, de l'azote et de l'oxygène sont observables en totalité. Cependant, comme les traces peuvent être identifiées non seulement par leur longueur mais aussi par leur diamètre (si celui-ci a une largeur suffisante), il est possible que les traces qui pénètrent dans le détecteur sous un angle de $90^{\circ}$ environ, même aux énergies plus faibles, soient observables.

Indépendamment de ces considérations, le nombre total théoriquement possible des trajectoires des ions de recul formés dans l'unité de surface du détecteur a été calculé.

Le nombre total des trajectoires possibles est donné par la relation :

où :

$$
N=\sigma \Phi A c
$$

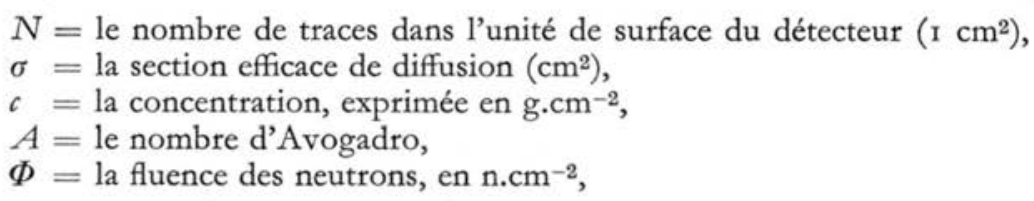

où :

$$
\begin{aligned}
& \Phi=\Phi_{0} \varphi\left(E_{n}\right), \\
& \sigma=\sigma\left(E_{n}\right),
\end{aligned}
$$

et :

$$
\begin{aligned}
& \varphi_{n}=\frac{n_{E_{n}}}{\int n_{E_{n}} \mathrm{~d} E_{n}}, n_{E_{n}} \text { est le nombre de neutrons d'énergie } E_{n}, \\
& \left.A c=N_{i} \text { (atome par } \mathrm{cm}^{2}\right) .
\end{aligned}
$$

Le nombre total de traces dues aux ions de recul est :

$$
\begin{aligned}
N\left(\frac{\text { traces }}{\mathrm{cm}^{2}}\right) & =\Phi_{0} \sum_{i=1}^{3} N_{i} \int_{E \min }^{E \max } \sigma_{e l_{i}}\left(E_{n}\right) \varphi\left(E_{n}\right) \mathrm{d} E_{n} \\
\bar{\sigma}_{e l_{i}} & =\int_{E \min }^{E \max } \sigma_{e l_{i}}\left(E_{n}\right) \varphi\left(E_{n}\right) \mathrm{d} E_{n}
\end{aligned}
$$

où $\bar{\sigma}_{e l_{i}}$ est la section moyenne de diffusion élastique sur le spectre des neutrons. En utilisant les sections efficaces de diffusion élastique de [10], [ $\mathrm{Ir}],[\mathrm{I} 2]$ et le spectre des neutrons émis par la source de Pu-Be que nous avons utilisée dans nos 
expériences [13], la relation (14) a été intégrée numériquement. Nous avons obtenu pour l'intervalle $E_{\min }-E_{\max }$, les valeurs moyennes des sections élastiques données ci-dessous. $E_{\max }$ est la limite supérieure de l'énergie du spectre de la source de $\mathrm{Pu}-\mathrm{Be}$ utilisée, et $E_{\min }$ a été choisie en raison des conditions d'observation des traces au microscope optique, [7], [8], (tableau I) :

$$
\begin{aligned}
& \bar{\sigma}_{e l_{C}}(5-\text { 10 MeV) }=0,96 \mathrm{~b} \\
& \sigma_{e l_{N}}(6-\text { 10 MeV) }=0,93 \mathrm{~b} \\
& \sigma_{e l_{O}}(8-\text { 10 MeV) }=0,73 \mathrm{~b}
\end{aligned}
$$

En fonction de la composition élémentaire du nitrate de cellulose donnée dans le tableau II, nous avons calculé les concentrations en atomes par $\mathrm{cm}^{2}$, en supposant une épaisseur active du détecteur $b$ (comme cible pour les neutrons) de $2 \mu \mathrm{m}$.

On a trouvé :

$$
N_{i}\left(\frac{\text { at. }}{\mathrm{cm}^{2}}\right)=N_{i}^{\prime}\left(\frac{\text { at. }}{\mathrm{cm}^{3}}\right) b(\mathrm{~cm})
$$

$$
\begin{aligned}
& N_{C}=3,82 \cdot 10^{18} \text { at.cm-2 } \\
& N_{N}=1,53 \cdot 10^{18} \text { at.cm-2 } \\
& N_{O}=6,89 \cdot 10^{18} \text { at.cm-2 }
\end{aligned}
$$

En substituant (I5) et (17) en (13), nous avons obtenu :

$$
\sum_{i=1}^{3} \bar{\sigma}_{e l_{N i}} \approx 10,12.10^{-6} \text { trace par neutron }
$$

En utilisant une valeur moyenne de $4 \cdot 10^{-8} \mathrm{rem} \cdot \mathrm{cm}^{2} \cdot \mathrm{n}^{-1}$, et une épaisseur utile du détecteur de $2 \mu \mathrm{m}$, nous avons trouvé pour les conditions ci-dessus une sensibilité de 3,95 mrem.cm² par trace.

Cette valeur est valable dans le cas où toutes les traces formées sont observables (ceci est discutable si on examine les parcours des ions dans le détecteur donnés dans le tableau I).

\subsection{RÉACtions $(n, \alpha)$ AVEC LES NOYAuX DE CARbone, Azote ET OXYGÈne}

Comme nous l'avons déjà mentionné, certains éléments constitutifs du nitrate de cellulose, donnent des réactions $(n, \alpha)$ avec les neutrons rapides.

En raison de l'utilisation de ce type d'interaction pour la dosimétrie des neutrons rapides, certains paramètres ont été examinés.

Les réactions $(n, \alpha)$ sur les noyaux légers, dans l'intervalle d'énergie de neutrons qui nous intéresse sont endoénergétiques; en effet, il y a un seuil d'énergie au-delà duquel la réaction est possible [4], [s].

Pour calculer l'énergie des particules $\alpha$ produites dans ces réactions, nous avons utilisé les relations $(1,2,3)$ qui deviennent :

$$
\begin{aligned}
& E_{n}+Q=E+E_{i} \\
& \sqrt{2 M_{n} E_{n}}=\sqrt{2 M_{\alpha} E_{\alpha}} \operatorname{Cos} \gamma+\sqrt{2 M_{i} N_{i}} \operatorname{Cos} \varphi \\
& \circ=\sqrt{2 M_{\alpha} E_{\alpha}} \operatorname{Sin} \gamma-\sqrt{2 M_{i} N_{i}} \operatorname{Sin} \varphi
\end{aligned}
$$

vOL. $7-\mathrm{N}_{2}$ 
où $Q$ est la chaleur de la réaction calculée par la relation :

$$
Q(\mathrm{MeV})=931,16\left(M_{n}+M_{a t}-M_{\alpha}-M_{i}\right) \quad \text { (u.a.m.) }
$$

et $M_{a t}=$ la masse de l'atome qui entre en réaction avec les neutrons.

Dans le tableau III sont données les réactions considérées, les chaleurs de réactions, et les énergies des seuils. Les énergies des seuils ont été calculés selon (4):

$$
\left(E_{n}\right)_{\text {seuil }}=-\mathcal{Q} \cdot\left(\frac{M_{n}+M_{a t}}{M_{a t}}\right)
$$

TABLEAU III

Chaleurs de réactions et ÉNERGies des Seuils de réactions $(n, \alpha)$

\begin{tabular}{c|c|c}
\hline \hline Réaction & $\mathcal{Q}(\mathrm{MeV})$ & Eseuil $(\mathrm{MeV})$ \\
\cline { 1 - 2 } \cline { 1 - 1 } & $-5,7080$ & 5,7560 \\
${ }_{6}^{12} \mathrm{C}+{ }_{0}^{1} n \rightarrow{ }_{2}^{4} \alpha+{ }_{4}^{9} \mathrm{Be}$ & $-0,1536$ & \\
${ }_{7}^{14} \mathrm{~N}+{ }_{0}^{1} n \rightarrow{ }_{2}^{4} \alpha+{ }_{5}^{11} \mathrm{~B}$ & & \\
${ }_{8}^{16} \mathrm{O}+{ }_{0}^{1} n \rightarrow{ }_{2}^{4} \alpha+{ }_{6}^{13} \mathrm{C}$ & $-2,2013$ & \\
\hline \hline
\end{tabular}

Les relations (1), (2), (3) ont été appliquées au cas des réactions $(n, \alpha)$ et, en considérant une valeur moyenne pour $E_{i}$, nous obtenons :

$$
\bar{E}_{i}=\frac{2 M_{n} M_{i}}{\left(M_{n}+M_{i}\right)^{2}}\left(E_{n}+Q\right)
$$

De (19) nous obtenons :

$$
\bar{E}_{\alpha}=\frac{M_{n}^{2}+M_{i}^{2}}{\left(M_{n}+M_{i}\right)^{2}}\left(E_{n}+\mathscr{Q}\right)
$$

Le tableau IV donne les énergies moyennes des particules obtenues par les réactions $(n, \alpha)$ considérées et calculées par les relations (22) et (23), les sections en millibarns et les parcours des particules $\alpha$ en fonction de l'énergie des neutrons.

Par un calcul analogue à celui fait pour les ions de recul, et l'emploi des relations (I 3 ), (I 8), nous obtenons le $\bar{\sigma}_{\alpha_{i}}$ sur le spectre des neutrons rapides de la source de Pu-Be utilisée dans les expériences.

$$
\begin{aligned}
& \bar{\sigma}_{\alpha_{C}}(6,5-\text { I0 } \mathrm{MeV})=39,21 \text { millibarns } \\
& \bar{\sigma}_{\alpha_{N}}(1,2-8,5 \mathrm{MeV})=164,18 \text { millibarns } \\
& \bar{\sigma}_{\alpha_{O}}(3,7-\text { I0 } \mathrm{MeV})=57,76 \text { millibarns }
\end{aligned}
$$

Il en résulte que la réaction ayant la section efficace moyenne la plus importante est ${ }_{7}^{14} \mathrm{~N}(n, \propto){ }_{5}^{11} \mathrm{~B}$. Cependant, la plus grande contribution est due à la réaction sur l'oxygène en raison de la concentration élevée de celui-ci dans le détecteur (relation 17). 


\section{TABLEAU IV}

Section efFicace des réactions $(n, \alpha)$ SUR les NOYAUX de C, N, O

ET PARCOURS DES PARTICULES $\propto$ OBTENUES DANS LE NITRATE DE CELLULOSE

\begin{tabular}{|c|c|c|c|c|c|c|c|c|c|c|c|c|}
\hline \multicolumn{5}{|c|}{ Carbone } & \multicolumn{4}{|c|}{ Azote } & \multicolumn{4}{|c|}{ Oxygène } \\
\hline $\begin{array}{c}E_{n} \\
(\mathrm{MeV})\end{array}$ & $\begin{array}{c}\bar{E}_{9_{B e}} \\
(\mathrm{MeV})\end{array}$ & $\begin{array}{c}\bar{E}_{\alpha} \\
(\mathrm{MeV})\end{array}$ & $\begin{array}{c}\mathrm{R}_{\alpha} \\
(\mu \mathrm{m})\end{array}$ & $\begin{array}{c}\sigma_{\alpha} \\
\text { m barn }\end{array}$ & $\begin{array}{r}\bar{E}_{11} B \\
(\mathrm{MeV})\end{array}$ & $\begin{array}{c}\bar{E}_{\alpha} \\
(\mathrm{MeV})\end{array}$ & $\begin{array}{c}R_{\alpha} \\
(\mu \mathrm{m})\end{array}$ & $\begin{array}{c}\sigma_{\alpha} \\
\text { mbarn }\end{array}$ & $\begin{array}{r}\bar{E}_{13} \\
(\mathrm{MeV})\end{array}$ & $\begin{array}{c}\bar{E}_{\boldsymbol{\alpha}} \\
(\mathrm{MeV})\end{array}$ & $\begin{array}{c}\mathrm{R}_{\alpha} \\
(\mu \mathrm{m}) \mathrm{m}\end{array}$ & $\begin{array}{l}\sigma_{\alpha} \\
\text { barn }\end{array}$ \\
\hline 1,0 & & & & & $0, \mathrm{I} \mathrm{I}$ & 0,74 & 2,36 & 0 & & & & \\
\hline $\mathrm{I}, \mathrm{I}$ & & & & & 0,12 & 0,83 & 2,63 & 0 & & & & \\
\hline 1,2 & & & & & 0,13 & 0,92 & 2,90 & Io & & & & \\
\hline 1,5 & & & & & 0,17 & $\mathrm{I}, \mathrm{I} 8$ & 3,75 & 20 & & & & \\
\hline 1,8 & & & & & 0,20 & 1,44 & 4,69 & 125 & & & & \\
\hline 2,0 & & & & & 0,23 & 1,62 & 5,37 & 35 & & & & \\
\hline 2,5 & & & & & 0,29 & 2,06 & 7,18 & Iso & & & & \\
\hline 3,0 & & & & & 0,35 & 2,49 & 9,09 & 230 & & & & ** \\
\hline 3,5 & & & & & 0,42 & 2,93 & 11,20 & 335 & 0,14 & 1,16 & 3,67 & 0 \\
\hline 4,0 & & & & & $0,4^{8}$ & 3,37 & 13,59 & 365 & 0,20 & 1,60 & 5,30 & 76 \\
\hline 4,5 & & & & & 0,54 & 3,81 & 16,27 & 280 & 0,26 & 2,04 & 7,13 & 34 \\
\hline 5,0 & & & & & 0,60 & 4,25 & 19,21 & 230 & 0,31 & 2,49 & 9,07 & 89 \\
\hline 5,5 & & & & & 0,66 & 4,68 & 22, II & 80 & 0,37 & 2,93 & I I , 2I & 3 \\
\hline 6,0 & 0,04 & 0,25 & 1,07 & $\mathbf{I}$ & 0,73 & 5,12 & 25,23 & 95 & 0,42 & 3,38 & 13,64 & 16 \\
\hline 7,0 & 0,18 & 1,11 & 3,52 & 10 & 0,85 & 6,00 & 32,56 & 40 & 0,53 & 4,27 & 19,34 & 63 \\
\hline 8,0 & 0,33 & 1,97 & 6,80 & 74 & 0,97 & 6,87 & 40,85 & 60 & 0,64 & 5,16 & 25,50 & so \\
\hline 9,0 & 0,47 & 2,83 & 10,66 & 59 & I, IO & 7,75 & 49, is & & 0,76 & 6,04 & $33, \mathrm{OI}$ & 71 \\
\hline 10,0 & $0,6 \mathrm{I}$ & 3,68 & 15,50 & 126 & 1,22 & 8,63 & 55,50 & & 0,87 & 6,93 & $4^{1,42}$ & 86 \\
\hline
\end{tabular}

** Au-dessous de $E_{n} \approx 3,65 \mathrm{MeV}, \sigma_{16} O(n, \alpha) \circ \approx[\mathrm{Ir}]$.

Le parcours moyen des particules $\alpha$ dans le nitrate de cellulose, en fonction de l'énergie des neutrons, a été calculé selon la méthode utilisée pour les ions de recul.

Des relations (1 8) avec $\mathrm{el}_{i} \rightarrow \alpha_{i}$ et (24), nous avons trouvé pour une épaisseur active de $20 \mu \mathrm{m}$ :

$$
\sum_{i=1}^{3} \bar{\sigma}_{\alpha_{i}} N_{i} \approx 7,99 \cdot 10^{-6} \text { trace par neutron }
$$

Il en résulte pour cette méthode (basée sur les traces $\alpha$ des réactions $(n, \alpha)$ ) une sensibilité de $5 \mathrm{~m}$ rem. $\mathrm{cm}^{2}$ par trace. Toutefois, cette valeur théorique contient une certaine part d'arbitraire, due à l'absence d'un critère objectif pour le choix de l'épaisseur de la couche active du détecteur qui joue le rôle de cible. Nous avons choisi pour cette épaisseur la valeur de $20 \mu \mathrm{m}$ en tenant compte du fait que la longueur moyenne des trajectoires $\alpha$ obtenues est de cet ordre de grandeur.

\subsection{RÉACtions MULTiples}

Pour des neutrons d'énergie plus élevée, il se produit des réactions avec production de plusieurs particules $\alpha$. Dans le cas du détecteur de nitrate de

vOL. $7-\mathrm{N}_{2}$ 
cellulose, les réactions suivantes peuvent nous intéresser pour la dosimétrie des neutrons :

\begin{tabular}{cll}
\multicolumn{1}{c}{ Réaction } & Chaleur de réaction & Energie de seuil \\
${ }^{12} \mathrm{C}\left(n, n^{\prime}\right) 3 \alpha$ & $\mathscr{Q}=-7,28 \mathrm{MeV}$ & $E_{s}=7,89 \mathrm{MeV}$ \\
${ }^{14} \mathrm{~N}(n, 2 \alpha){ }^{7} \mathrm{Li}$ & $\mathscr{Q}=-8,82 \mathrm{MeV}$ & $E_{s}=9,45 \mathrm{MeV}$ \\
${ }^{14} \mathrm{~N}(n, t){ }_{3} \alpha$ & $\mathscr{Q}=-11,29 \mathrm{MeV}$ & $E_{s}=12,2 \mathrm{MeV}$ \\
${ }^{16} \mathrm{O}\left(n, n^{\prime}\right){ }_{4} \alpha$ & $Q=-14,50 \mathrm{MeV}$ & $E_{s}=15,4 \mathrm{IMeV}$
\end{tabular}

Pour les énergies de neutrons inférieures à ro $\mathrm{MeV}$, la contribution de ce type de réactions n'est pas importante, et elles n'ont pas été prises en considération dans ce travail.

\subsection{Sensibilití théorique globale}

L'addition des nombres de traces des ions de recul et des traces dues aux réactions $(n, \alpha)(18),(25)$ nous donne :

$$
\sum_{i=1}^{3} N_{i} \bar{\sigma}_{e l_{i}}+\sum_{i=1}^{3} N_{i} \bar{\sigma}_{\alpha i} \approx 18,11 \cdot 10^{-6} \text { trace par neutron }
$$

De (27) il résulte une sensibilité générale de 2,21 mrem. $\mathrm{cm}^{2}$ par trace.

Dans le cas où l'efficacité de l'enregistrement du détecteur pour ces deux types de traces (ions de recul et particules $\alpha$ ) serait de 100 p. 100, le rapport des nombres de traces d'ions de recul aux traces de particules $\alpha$ formées par les réactions $(n, \alpha)$ serait de :

$$
\frac{N_{\text {ion }}}{N_{\alpha}} \approx \text { 1,27. }
$$

Cependant, ce rapport théorique doit être corrigé de l'efficacité d'enregistrement des traces $\alpha$ dans le détecteur qui varie en fonction de l'énergie des particules $\alpha$ ainsi que de la présence des traces $\alpha$ obtenues dans les réactions de diffusion multiple, qui n'ont pas été prises en considération.

\section{4 - CARACTÉRISTIQUES DU DÉTECTEUR DE NITRATE DE CELLULOSE}

Le détecteur de nitrate de cellulose a été obtenu par coulée sur verre du collodion commercial à l'acétate d'amyle. La technique de préparation du détecteur et ses caractéristiques d'enregistrement ont été présentées en détail dans deux autres travaux antérieurs [9] [14]. En accord avec les données présentées ici, pour augmenter la reproductibilité du détecteur et le rapport $V_{t} / V_{m}\left(V_{t}=\right.$ vitesse d'attaque le long de la trace et $V_{m}=$ vitesse d'attaque en masse du détecteur), les feuilles de nitrate de cellulose ont été chauffées avant l'emploi pendant 2 à 4 heures à la température de $110^{\circ} \mathrm{C}$. Le traitement des détecteurs a été effectué 
à la température de $60^{\circ} \mathrm{C}$ pendant 20 minutes ou à $70^{\circ} \mathrm{C}$ pendant 10 minutes dans de la soude à 20 p. 100 . Pour le détecteur ainsi traité, on a déterminé une énergie critique d'enregistrement des traces $\alpha$ de $4,8 \mathrm{MeV}$.

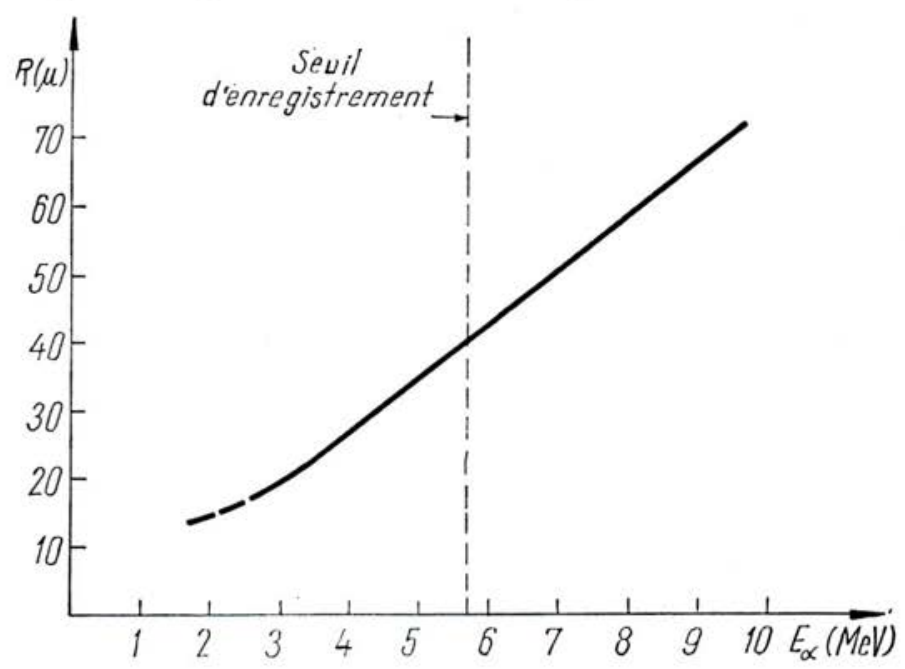

Fig. I. - Courbe parcours-énergie pour les particules $\alpha$ dans le nitrate de cellulose.

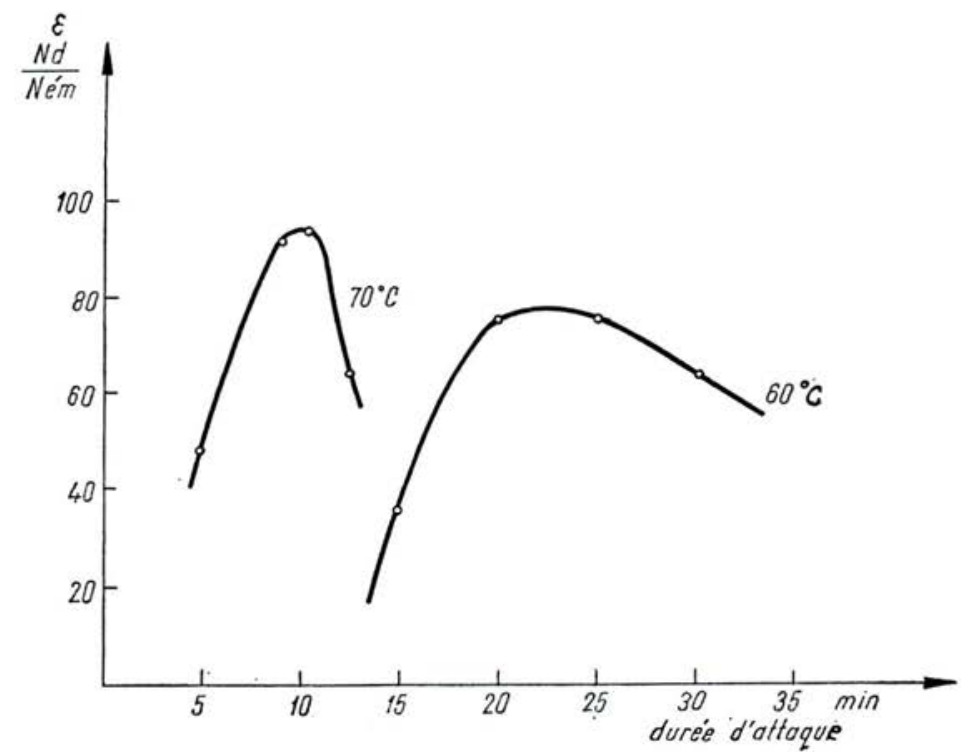

Fig. 2. - Variation de l'efficacité d'enregistrement en fonction de conditions d'attaque pour les particules $\alpha$ de ${ }^{241} \mathrm{Am}\left(E_{\alpha}=5,48 \mathrm{MeV}\right)$.

vOL. $7-\mathrm{N}_{2}$ 
Dans la figure $\mathrm{I}$, on donne les courbes des relations parcours-énergie pour les particules $\alpha$ dans le nitrate de cellulose. Sur ce graphique, la ligne verticale marque la limite de l'intervalle d'énergie au-delà duquel le parcours des particules $\alpha$ est enregistré intégralement dans le détecteur. La figure 2 représente l'efficacité d'enregistrement des traces $\alpha$ de l'américium 24I dans le détecteur de nitrate de cellulose en fonction des conditions du traitement. Pour des particules $\alpha$ de $5,5 \mathrm{MeV}$, l'efficacité d'enregistrement se rapproche de $100 \mathrm{p} .100$, à condition que l'attaque chimique soit effectuée à $70^{\circ} \mathrm{C}$.

Une autre caractéristique importante du détecteur est sa stabilité. De ce point de vue, nous avons étudié la variation de la sensibilité du détecteur de nitrate de cellulose utilisé jusqu'à 3 mois après sa préparation, ainsi que la régression des traces enregistrées pour différentes températures et durées. Les résultats présentés dans la figure 3 montrent que la sensibilité du détecteur ne varie pas d'une manière significative sur une durée de 75 jours après sa préparation.

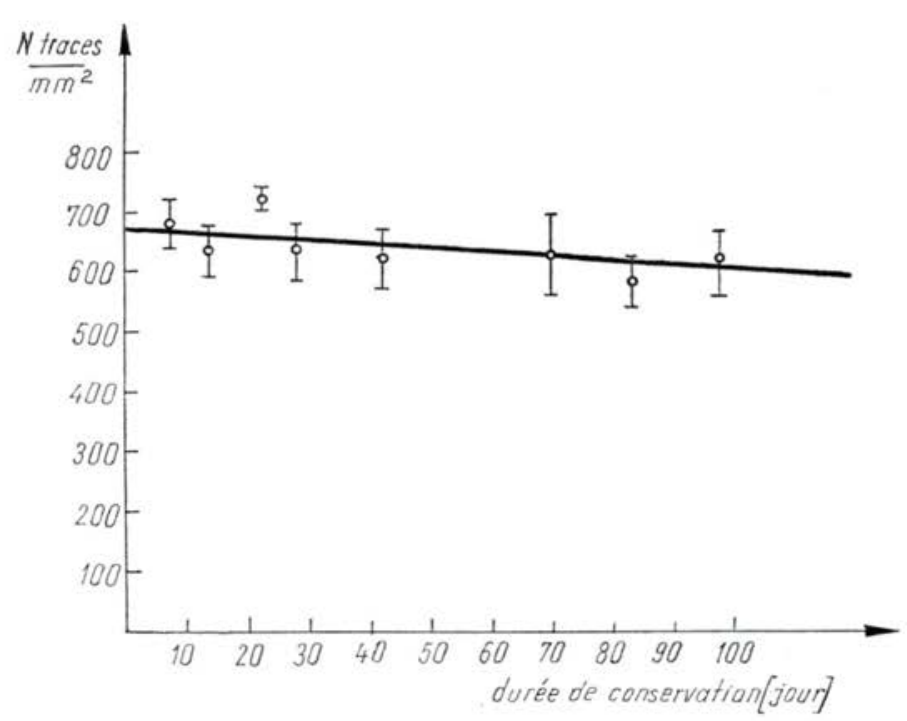

FIG. 3. - Variation de la sensibilité du détecteur de nitrate de cellulose au cours de sa conservation.

On a constaté également que, jusqu'à une température de so à $70^{\circ} \mathrm{C}$, les traces sont très stables. La densité de traces reste pratiquement constante quand le détecteur est conservé pendant 60 jours à la température ambiante avant attaque.

\section{s - RÉSULTATS EXPÉRIMENTAUX}

Dans le but de vérifier la concordance des résultats théoriques, plusieurs séries de détecteurs de nitrate de cellulose ont été exposées à une source de 
neutrons de $\mathrm{Pu}-\mathrm{Be}$ ayant une fluence connue de neutrons. Sur les feuilles de détecteurs exposées et développées, par observation au microscope optique, on a déterminé la densité des traces $\alpha$ et celle des ions de recul. Certains résultats sont donnés dans le tableau V. L'exploration de ces détecteurs nous a montré que la densité des traces dues aux ions de recul est légèrement différente de celle calculée théoriquement (soit supérieure, soit inférieure) ce qui s'explique par le fait que les traces d'ions sont plus difficilement visualisées. Au contraire, les traces $\alpha$ sont plus facilement mises en évidence en raison de leur forme allongée et donc plus faciles à compter.

\section{TABLEAU $\mathrm{V}$}

Résultats expérimentaux SUR LA fluence D'UNe SOURCE DE NeUtrons RApides Pu-Be, DÉTERMINÉE PAR LA MÉTHODE PRÉSENTÉE

\begin{tabular}{|c|c|c|c|c|c|}
\hline \multirow{2}{*}{$\begin{array}{c}\Phi^{*} \text { calc. } \\
\mathrm{n} / \mathrm{cm}^{2}\end{array}$} & & \multicolumn{3}{|c|}{$\Phi$ mesuré $\mathrm{n} / \mathrm{cm}^{2}$} & \multirow{2}{*}{$\bar{\Phi}$ mes. $\mathrm{n} / \mathrm{cm}^{2}$} \\
\hline & I & 2 & 3 & 4 & \\
\hline I. $1,73 \cdot 10^{8}$ & $1,146.10^{8}$ & $1,077 \cdot 10^{8}$ & I, I IO. & - & $\mathrm{I}, \mathrm{III} \pm 0,028 \cdot 10^{8}$ \\
\hline II. $7,54 \cdot 10^{9}$ & $5,734 \cdot 10^{9}$ & $5,306 \cdot 10^{9}$ & $5,725 \cdot 10^{9}$ & $5,250.10^{9}$ & $5,501 \pm 0,225 \cdot 10^{8}$ \\
\hline III. $7,54 \cdot 10^{9}$ & $4,60.10^{9}$ & $6,96 \cdot 10^{9}$ & $5,5 \times 3 \cdot 10^{9}$ & $5,775 \cdot 10^{9}$ & $5,705 \pm 0,960 \cdot 10^{9}$ \\
\hline IV. $4,51.10^{9}$ & $3,905 \cdot 10^{9}$ & $3,552.10^{9}$ & $3,345 \cdot 10^{9}$ & $3,295 \cdot 10^{9}$ & $3,524 \pm 0,241 \cdot 10^{9}$ \\
\hline V. $4,51.10^{9}$ & $3,996 \cdot 10^{9}$ & $3,510.10^{9}$ & $3,221 \cdot 10^{9}$ & - & $3,575 \pm 0,320 \cdot 10^{9}$ \\
\hline
\end{tabular}

* $\Phi$ a été calculé à partir de l'activité donnée sur le certificat d'étalonnage de la source.

Des résultats du tableau $\mathrm{V}$, il résulte que le nombre des traces $\alpha$ est légèrement inférieur à la valeur théorique, ce qui peut être expliqué par les raisons suivantes :

a) La possibilité de confondre les traces $\alpha$ ayant une incidence normale avec les ions de recul;

b) La dépendance de l'efficacité de l'enregistrement du détecteur vis à vis de l'énergie de la particule $\alpha$.

Pour prendre en considération la contribution du deuxième facteur, nous avons introduit un facteur de correction, $k_{2}$, dans la formule de la dose :

$$
D=k_{1} k_{2} N
$$

$N=$ nombre de traces $\alpha$ par $\mathrm{cm}^{2}$ dans le détecteur, déterminé par observation au microscope optique,

$k_{1}=$ facteur de qualité calculé en nombre de neutrons par particule $\alpha$,

$k_{2}=$ efficacité d'enregistrement des traces $\alpha$ dans le nitrate de cellulose en fonction de l'énergie des particules $\alpha$, donc de l'énergie des neutrons.

vOL. $7-\mathrm{N}^{\circ}$ 
Selon certaines données de la littérature et de nos travaux, cette dépendance de l'efficacité d'enregistrement en fonction de l'énergie des particules $\alpha$ a approximativement la forme de la courbe représentée dans la figure 4 .

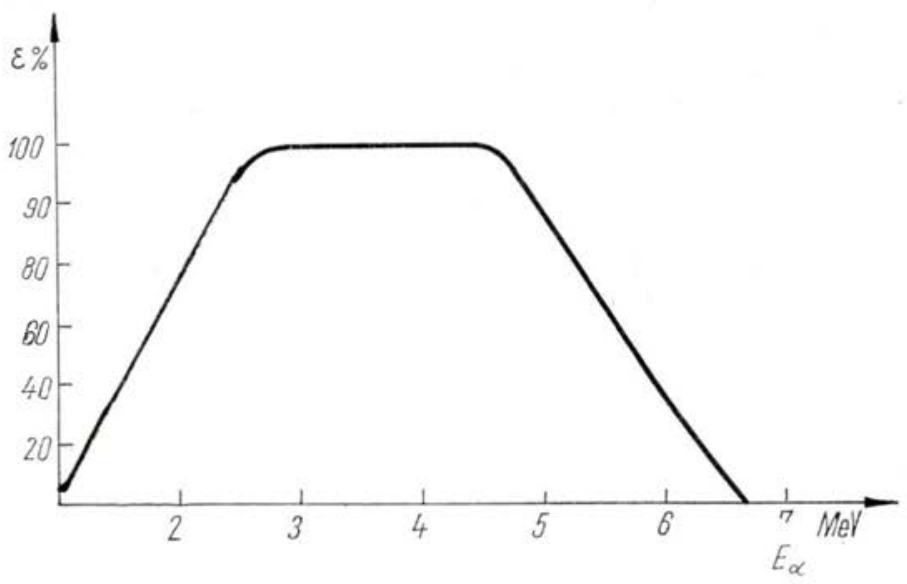

Fig. 4. - Dépendance de l'efficacité d'enregistrement des traces $\alpha$ dans le détecteur de nitrate de cellulose en fonction de l'énergie.

Dans l'intervalle d'énergie de $\mathrm{I}$ à ro $\mathrm{MeV}, k_{1}$ varie légèrement avec l'énergie des neutrons. Dans ce cas, nous avons utilisé la valeur moyenne de $4.10^{-8}$ rem. $n^{-1} \cdot \mathrm{cm}^{2}$.

La dose de neutrons rapides devient dans ce cas :

$$
D=s \text { (mrem.cm² par trace) } k_{2} N
$$

\section{6 - CONCLUSION}

Les considérations théoriques et nos résultats expérimentaux nous ont permis d'établir les conclusions suivantes :

I) Le détecteur de nitrate de cellulose peut être utilisé en principe pour la dosimétrie des neutrons rapides en utilisant deux types d'interactions des neutrons avec les atomes du détecteur : diffusion élastique sur les noyaux de $\mathrm{C}, \mathrm{N}, \mathrm{O}$, et réaction $(n, \alpha)$ avec les mêmes noyaux;

2) En raison de certaines difficultés expérimentales relatives à la longueur très réduite des parcours des noyaux de recul pour les énergies de neutrons inférieures à $6 \mathrm{MeV}$, la méthode des noyaux de recul n'est utilisable pour la dosimétrie des neutrons rapides que pour les hautes énergies, au-delà de 6 à $7 \mathrm{MeV}$. Pour éviter les erreurs de mesure dues à la présence des traces $\alpha$, nous estimons que, au cas où la dosimétrie des neutrons de hautes énergies est faite 
à partir des traces d'ions de recul, il est souhaitable d'utiliser comme détecteur le polycarbonate, qui est moins sensible aux particules $\alpha$ que le nitrate de cellulose;

3) Nous estimons que la méthode la plus convenable pour la dosimétrie de neutrons rapides dans l'intervalle d'énergie de 2-10 $\mathrm{MeV}$, avec le nitrate de cellulose est celle basée sur les réactions $(n, \alpha)$ sur les noyaux de $\mathrm{C}, \mathrm{N}, \mathrm{O}$. Selon les calculs présentés dans ce travail, la réaction ${ }_{8}^{16} \mathrm{O}(n, \alpha){ }_{8}^{13} \mathrm{C}$ a la contribution la plus importante de ce point de vue. En supposant que l'efficacité d'enregistrement des traces $\alpha$ dans le détecteur, dans cet intervalle d'énergie de neutrons, est approximativement de $100 \mathrm{p}$. I00, la sensibilité de la méthode est de $7,99 \cdot 10^{-6}$ trace par neutron, donc de s mrem.cm² par trace.

Les traces $\alpha$ obtenues dans ces réactions et enregistrées par le détecteur sont très facilement mises en évidence et comptées au microscope optique. On obtient une concordance assez bonne entre les données théoriques sur la sensibilité de la méthode et les résultats expérimentaux.

Aux énergies plus élevées des neutrons, donc des particules $\alpha$ obtenues, l'efficacité d'enregistrement du détecteur diminue beaucoup et l'utilisation de la méthode est limitée. De plus, pour les énergies de neutrons supérieures à $8 \mathrm{MeV}$, des réactions du type $(n, 2 \alpha),(n, 3 \alpha),(n, 4 \alpha)$ sont probables. Nous aborderons le problème de ces réactions dans un travail ultérieur.

Nous estimons cependant que, pour les cas usuels de la dosimétrie des neutrons rapides auprès des sources de neutrons de $\mathrm{Ra}-\mathrm{Be}, \mathrm{Po}-\mathrm{Be}, \mathrm{Pu}-\mathrm{Be}$, où la limite supérieure du spectre d'énergie se situe à Io-I $\mathrm{MeV}$, et auprès des réacteurs, la méthode basée sur les réactions $(n, \alpha)$ dans le nitrate de cellulose est utilisable.

4) Par comparaison avec les émulsions nucléaires, la méthode des détecteurs solides (nitrate de cellulose) pour la dosimétrie des neutrons rapides, est plus commode et plus simple à utiliser. Les traces enregistrées ne sont pas affectées par les facteurs environnants et les détecteurs peuvent être conservés plusieurs mois à température ambiante sans noter de variation de la densité des traces enregistrées. La régression des traces lors de l'emploi du dosimètre personnel ne sera donc pas significative.

En ce qui concerne la précision de la méthode, celle-ci est affectée par :

I) La dépendance de la section efficace de réaction vis-à-vis de l'énergie des neutrons, section qui est insuffisamment connue pour cet intervalle d'énergie;

2) La dépendance de l'efficacité de l'enregistrement des particules $\alpha$ dans le détecteur vis-à-vis de l'énergie des particules $\alpha$, pour les énergies supérieures à s $\mathrm{MeV}-$ s,s $\mathrm{MeV}$.

Ces deux facteurs sont les plus importants. Ils introduisent des imprécisions dans le calcul de la dose, en fonction de la densité de traces mesurée dans le détecteur. Le premier facteur est commun aux émulsions nucléaires et aux détecteurs solides, mais le second est propre aux détecteurs solides.

La méthode étudiée a donc trois avantages par rapport à la méthode des émulsions nucléaires pour la dosimétrie des neutrons rapides : simplicité et commodité dans l'exploitation, stabilité du détecteur, stabilité des traces enregistrées. Mais elle présente aussi des inconvénients importants : sensibilité plus réduite, dépendance de l'efficacité d'enregistrement du détecteur vis-à-vis de l'énergie des particules $\alpha$ au-delà d'une certaine énergie (correspondant aux

VOL. $7-\mathrm{N}^{\circ} 2$ 
neutrons d'énergie supérieure à ro $\mathrm{MeV}$ ), existence d'un seuil de l'énergie des neutrons au-delà duquel la méthode peut être utilisée. Ce seuil se situe environ à $2 \mathrm{MeV}$ alors qu'il est de $500 \mathrm{keV}$ pour les émulsions nucléaires.

Cependant, en raison de l'insensibilité du détecteur solide aux rayons $\gamma$ et aux électrons, cette méthode s'avère plus utile que celle des émulsions nucléaires. La précision sur la dose de neutrons rapides pourrait être meilleure par l'emploi du détecteur de nitrate de cellulose, à condition que le détecteur soit utilisé pour la dosimétrie des neutrons compris dans l'intervalle d'énergie pour lequel il a été étalonné.

\section{RÉFÉRENCES}

[1] Mosinski G. Contribution à l'étude de l'enregistrement des traces dans les détecteurs solides visuels. Application à l'étude de la dosimétrie des neutrons rapides. Thèse de doctorat, $3^{\mathrm{e}}$ cycle, Strasbourg, 24 Oct. 1968.

[2] TuYn J.W.N., Broerse J.J. Analysis of the etch pit size distribution in Makrofol polycarbonate. $7^{t h}$ Conf. on Nuclear Pbotograpby, Barcelona 1970.

[3] Turn J.W.N., Broerse J.J. On the use of Makrofol polycarbonate foils for the measurements of the fast neutron dose distribution inside a human phantom. $7_{\text {th }}$ Conf. on Nuclear Pbotography, Barcelona 1970.

[4] Evans R.D. The atomic nucleus. Mc. Graw-Hill, igss.

[s] SEgré E. Nuclear experimental physics. Vol. II. John Wiley \& Sons, New York. Chapman \& Hall, Limited, London.

[6] Heckman H.H., Perkins B.L., Simon W.S., Smith F.M., Barkas W.H. Pbys. Rev., i17, 2, 544 (1960).

[7] Fleischer R.L., Price P.B., Walker R.M., Hubbard E.L. Pbys. Rev., 133, sA (1964).

[8] Ralarosy J., Schmidt R., Mosinski G., Tripier J., Debeauvais M., Stein R., Remy G. Journal de Pbys. T. 30, I, p. I (1969).

[9] Nicolae M. Rev. Roum. de Phys., is p., 881-889, (1970).

[io] Schmidt J.J. KFK I20 (1962).

[ii] LANGer I., SChmidt J.J., Woll D. KFK 750 (i968).

[12] BNL 325, Vol. I (1964).

[13] Pannetier R. Vade-Mecum du technicien, Tome I, (1965).

[14] Nicolae M. Rev. Roum. Phys. sous presse. 